\title{
基于 OTSUKF 的飞行器惯性测量单元的故障诊断
}

\author{
何启志 ${ }^{1}$, 章卫国 ${ }^{1}$, 黄得刚 ${ }^{2}$, 陈华坤 ${ }^{1}$ ，刘璟龙 ${ }^{1}$
}

(1.西北工业大学 自动化学院, 陕西 西安 710129; 2. 兵器工业集团 航空弹药研究院, 黑龙江 哈尔滨 150036)

\begin{abstract}
摘 要: 线性系统存在随机偏差情况下, 最优二步卡尔曼滤波( OTSKF) 可以获得系统状态及偏差的最 优估计。无迹卡尔曼滤波( UKF) 利用 Sigma 点采样和 UT 变换技术经非线性系统传播状态的均值和 方差, 是一种典型的非线性滤波方法。飞行器是一种典型复杂非线性系统, 将惯性测量单元( IMU) 的 故障作为一种随机偏差处理,建立了包含 IMU 故障的滤波模型。将 UKF 算法和二步滤波思想应用到 飞行器之中,提出了一种适用于飞行器 IMU 故障诊断的最优二步无迹卡尔曼滤波( OTSUKF) 算法。 针对于飞行器,提出了一种滤波模型设计的方法。基于该模型,应用所提出的 OTSUKF 算法实现了飞 行器状态的最优估计和 IMU 故障的辨识,该算法经过了实际飞行数据验证其对风扰动具有鲁棒性并 且与已经被提出的迭代最优二步扩展卡尔曼滤波(IOTSEKF) 方法进行了对比验证其最优性。
\end{abstract}

\section{关 键 词: 最优二步无迹卡尔曼滤波; 随机偏差; 惯性测量单元; 状态估计; 故障诊断 \\ 中图分类号: V240.2 \\ 文献标志码:A \\ 文章编号 : 1000-2758(2018) 05-0933-09}

惯性测量单元 (IMU) 故障已经成为当前军机、 民机以及无人机飞行事故的一大主要原因。1995 年, Dryden 飞行研究中心对 Perseus 飞行事故进行 分析, 确定事故原因为: 俯仰角速度传感器故障导致 飞行速度超出正常范围限制, 机翼承受过大气动力 而发生折断。2005 年, MH 124 客机飞行过程中遭 遇主飞行仪表数据显示矛盾、自动右转向、自动超高 度飞行等一系列问题, 飞行事故原因确定为大气数 据惯性基准组件中的加速度传感器故障。

现阶段国内外飞行器主要基于硬件余度和表决 检测来实现惯性测量单元的容错。惯性测量单元常 见的余度配置方案包括正交配置和斜置配置 2 种， Honeywell 公司的 ARINC704 惯性基准系统, 采用正 交配置和表决监测实现惯性测量单元的容错, 波音 公司的 Boing-777 采用斜置配置及广义似然比检验 方法实现惯性测量单元的容错。硬件余度方案可以 大幅度提升飞行器关键传感器的容错能力, 但是也 具有成本较高及容易发生共性故障的缺点。例如, 虽然飞控系统关键传感器采用多余度配置, 但是自 2003 年起, 仅大气数据传感器故障引发的民机飞行 事故多达 35 起。这些事故的典型的代表有 2008 年
美国 B-2 轰炸机坠毁, 2009 年法航 447 航班事故都 是由于飞控系统关键传感器共性故障引起的。共性 故障导致飞行事故的原因是传感器硬件配置的相似 余度, 如果进行非相似余度配置, 可以进一步提升飞 行器的容错能力。解析余度的引人一直是飞行器传 感器容错中的热点问题, 它的引人可以补充硬件余 度的不足。

解析余度技术依赖于飞行器的动力学和运动学 模型, 飞行器的动力学模型和运动学模型以微分方 程表达, 通过对状态微分方程的积分可以得到传感 器容错所需要的状态信息。但是传感器的噪声及故 障是广泛存在的, 随着时间的积累, 传感器噪声及故 障信息经过积分环节会导致状态信息不可用, 这也 是解析余度应用于传感器容错的主要问题。传统的 非线性卡尔曼滤波算法,如扩展卡尔曼滤波( EKF)、 无迹卡尔曼滤波 (UKF) 等, 可以实现噪声存在情况 下飞行器的状态估计, 但是当传感器故障存在的情 况下,传统的非线性卡尔曼滤波算法的状态估计不 准确, 若故障幅值较大甚至导致状态信息不可用。

1967 年, Friedland 提出了二步卡尔曼滤波 ( TSKF) , 这种滤波器针对常值偏差可以保证估计的 
最优性 ${ }^{[1]}$ 。1993 年, Alouani 阐述了在随机偏差条 件下二步卡尔曼滤波的无偏差滤波器的误差协方差 矩阵满足某个代数条件限制的情况下, 等价于增广 状态卡尔曼滤波 (ASKF), 即保证了状态估计的最 优性, 但该约束条件在实际系统往往是不成立 的 ${ }^{[2]}$ 。1999 年, Hesieh 和 Chen 提出了最优二步卡 尔曼滤波 (OTSKF), 去除了该约束条件, OTSKF 在 随机偏差存在情况下, 仍然可以保证滤波器状态估 计的最优性 ${ }^{[3]}$ 。文献 [4] 应用了二步扩展卡尔曼滤 波 (TSEKF) 实现了定常风场下的大气数据系统关 键传感器的信号融合与重构, 但文章中假设是定场 风场, 对于非定场风场情况, 无法保证最优性。本文 提出了最优二步无迹卡尔曼滤波 (OTSUKF), 实现 了非定场风场下飞行器的状态最优估计及惯性量测 量传感器的故障辨识, 并且以实际飞行数据进行了 验证。

\section{1 问题描述}

IMU 是飞行控制系统关键传感器, 当 IMU 发生 故障时,将会导致灾难性的后果。为了保证传感器 数据的可靠性,工程中常为传感器采用硬件多余度 配置, 但硬件余度会使成本增加, 负荷增加且容易发 生共性故障等缺点。如何利用解析余度技术实现飞 行器 IMU 的故障诊断是本文研究的问题。

\section{2 滤波模型的建立}

本文的研究对象是飞控系统关键传感器,利用 卡尔曼滤波实现 IMU 的故障诊断。卡尔曼滤波基 于解析余度, 解析信号的精度取决于很大程度取决 于模型的准确性。文献 $[5]$ 给出了飞行器的力学 方程：

$$
\left\{\begin{array}{l}
\dot{u}=v r-w q-g \sin \theta+\frac{1}{m}(X+T) \\
\dot{v}=-u r+w p+g \cos \theta \sin \varphi+\frac{1}{m} Y \\
\dot{w}=u q-v p+g \cos \theta \cos \varphi+\frac{1}{m} Z
\end{array}\right.
$$

式中, $T$ 表示发动机推力, $m$ 表示飞行器的质量, $g$ 表 示重力加速度, $u, v, w$ 表示地速在机体系的投影, $p$, $q, r$ 表示飞行器运动的三轴角速率, $\varphi, \theta, \psi$ 表示飞行 器的姿态角, $X, Y, Z$ 是飞行器所受的气动力在飞行
器机体系的投影, 它的准确程度依赖于气动导数 $C_{X}, C_{Y}, C_{z}$ 的准确程度:

$$
\left\{\begin{array}{l}
X=\bar{q} S C_{X}(\alpha, \beta, p, q, r, \delta, \cdots) \\
Y=\bar{q} S C_{Y}(\alpha, \beta, p, q, r, \delta, \cdots) \\
Z=\bar{q} S C_{Z}(\alpha, \beta, p, q, r, \delta, \cdots)
\end{array}\right.
$$

式中, $\bar{q}$ 表示动压, $S$ 表示机翼参考面积, $\alpha$ 表示迎 角, $\beta$ 表示侧滑角, $\delta$ 表示舵偏量。然而气动导数的 获取一般通过 CFD 计算或者风洞实验获取,在调用 模型的过程中,气动系数的获取一般根据多维插值 获得,存在较大的不确定性,不确定性会导致基于该 模型的滤波器精度下降, 较大幅度的不确定性甚至 会导致滤波发散与中止。

加速度计测量的是载体相对惯性空间的绝对加 速度和引力加速度之和, 称作 “比力”, 用符号 $\bar{f}$ 表 示,根据比力的定义:

$$
\left\{\begin{array}{l}
\bar{f}_{x}=\frac{1}{m}(X+T) \\
\bar{f}_{y}=\frac{1}{m} Y \\
\bar{f}_{z}=\frac{1}{m} Z
\end{array}\right.
$$

将公式(3)带人公式(1), 得到修改后的飞行器力学 方程：

$$
\left\{\begin{array}{l}
\dot{u}=v r-w q-g \sin \theta+\bar{f}_{x} \\
\dot{v}=-u r+w p+g \cos \theta \sin \varphi+\bar{f}_{y} \\
\dot{w}=u q-v p+g \cos \theta \cos \varphi+\bar{f}_{z}
\end{array}\right.
$$

文献 $[6]$ 给出了飞行器的运动学方程：

$$
\left\{\begin{array}{l}
\dot{\varphi}=p+(r \cos \varphi+q \sin \varphi) \tan \theta \\
\dot{\theta}=q \cos \varphi-r \sin \varphi \\
\dot{\psi}=q \frac{\sin \varphi}{\cos \theta}+r \frac{\cos \varphi}{\cos \theta}
\end{array}\right.
$$

考虑到加速度计和陀螺仪的测量噪声, 可得到:

$$
\left\{\begin{array}{l}
f_{x}=a_{x m}-f_{a x}-\omega_{a x} \\
\bar{f}_{y}=a_{y m}-f_{a y}-\omega_{a y} \\
\bar{f}_{z}=a_{z m}-f_{a z}-\omega_{a z} \\
p=p_{m}-f_{p}-\omega_{p} \\
q=q_{m}-f_{q}-\omega_{q} \\
r=r_{m}-f_{r}-\omega_{r}
\end{array}\right.
$$

式中, $a_{x m}, a_{y m}, a_{z m}$ 是加速度计的测量值, $p_{m}, q_{m}, r_{m}$ 是陀螺仪的测量值, $f_{a x}, f_{a y}, f_{a z}$ 是加速度计故障, $f_{p}$, $f_{q}, f_{r}$ 是陀螺仪故障, $\omega_{a x}, \omega_{a y}, \omega_{a z}$ 是加速度计测量噪 
声, $\omega_{p}, \omega_{q}, \omega_{r}$ 是陀螺仪测量噪声。将公式 (6) 代人 修改后的力学方程 (4) 和运动学方程 (5), 得到滤 波器的系统方程:

$$
\left\{\begin{aligned}
& \dot{u}= v\left(r_{m}-f_{r}-\omega_{r}\right)-w\left(q_{m}-f_{q}-\omega_{q}\right)- \\
& g \sin \theta+\left(a_{x m}-f_{a x}-\omega_{a x}\right) \\
& \dot{v}=-u\left(r_{m}-f_{r}-\omega_{r}\right)+w\left(p_{m}-f_{p}-\omega_{p}\right)+ \\
& g \cos \theta \sin \varphi+\left(a_{y m}-f_{a y}-\omega_{a y}\right) \\
& \dot{w}= u\left(q_{m}-f_{q}-\omega_{q}\right)-v\left(p_{m}-f_{p}-\omega_{p}\right)+ \\
& g \cos \theta \cos \varphi+\left(a_{z m}-f_{a z}-\omega_{a z}\right) \\
& \dot{\varphi}=\left(p_{m}-f_{p}-\omega_{p}\right)+\left[\left(r_{m}-f_{r}-\omega_{r}\right) \cos \varphi+\right. \\
&\left.\left(q_{m}-f_{q}-\omega_{q}\right) \sin \varphi\right] \tan \theta \\
& \dot{\theta}=\left(q_{m}-f_{q}-\omega_{q}\right) \cos \varphi-\left(r_{m}-f_{r}-\omega_{r}\right) \sin \varphi \\
& \dot{\psi}=\left(q_{m}-f_{q}-\omega_{q}\right) \frac{\sin \varphi}{\cos \theta}+ \\
&\left(r_{m}-f_{r}-\omega_{r}\right) \frac{\cos \varphi}{\cos \theta}
\end{aligned}\right.
$$

三天线 GNSS 可以输出飞行器的速度和姿态信 息 ${ }^{[7]}$, 可得到滤波器的观测方程:

$$
\left\{\begin{array}{l}
{\left[\begin{array}{l}
u_{G S m} \\
v_{G S m} \\
w_{G S m}
\end{array}\right]=\boldsymbol{R}_{\mathrm{DCM}}^{\mathrm{T}}\left[\begin{array}{l}
u \\
v \\
w
\end{array}\right]+\left[\begin{array}{l}
\nu_{u} \\
\nu_{v} \\
\nu_{w}
\end{array}\right]} \\
\varphi_{m}=\varphi+\nu_{\varphi} \\
\theta_{m}=\theta+\nu_{\theta} \\
\psi_{m}=\psi+\nu_{\psi}
\end{array}\right.
$$

式中, $u_{G S m}, v_{G S m}, w_{G S m}$ 是 GNSS 输出的导航坐标系的 飞行器速度信息, $\varphi_{m}, \theta_{m}, \psi_{m}$ 是 GNSS 输出的姿态信 息, $\nu_{u}, \nu_{v}, \nu_{w}$ 是 GNSS 输出速度信息的测量噪声, $\nu_{\varphi}, \nu_{\theta}, \nu_{\psi}$ 是 GNSS 输出姿态信息的测量噪声, DCM 是方向余弦矩阵, 它建立起了机体系与导航系的坐 标转换关系, 它的定义为:

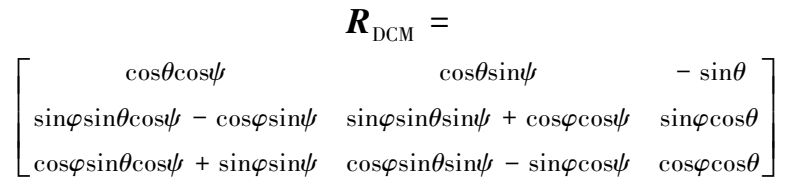

状态向量定义为: $\boldsymbol{x}=\left[\begin{array}{llll}u & v & w & \boldsymbol{\theta}\end{array}\right]^{\mathrm{T}}$, 观测向量定 义为: $\boldsymbol{y}=\left[u_{G S m} v_{G S m} w_{G S m} \varphi_{m} \theta_{m} \psi_{m}\right]^{\mathrm{T}}$, 输人向量定义 为: $\boldsymbol{u}=\left[a_{x m} a_{x m} a_{x m} p_{m} q_{m} r_{m}\right]^{\mathrm{T}}$, IMU 故障向量定义为: $\boldsymbol{f}=\left[f_{a x} f_{a x} f_{a x} f_{p} f_{q} f_{r}\right]^{\mathrm{T}}$ 。滤波模型可以简写成:

$$
\left\{\begin{array}{l}
\dot{\boldsymbol{x}}(t)=f(\boldsymbol{x}(t), \boldsymbol{u}(t), \boldsymbol{f}(t), \boldsymbol{\omega}(t)) \\
\boldsymbol{y}(t)=h(\boldsymbol{x}(t), \boldsymbol{\nu}(t))
\end{array}\right.
$$

线性化和离散化方法可参照文献 [4], 经过线性化 和离散化可以得到:

$$
\left\{\begin{array}{l}
\boldsymbol{x}_{k+1}=\boldsymbol{\Phi}_{k} \boldsymbol{x}_{k}+\boldsymbol{B}_{k} \boldsymbol{u}_{k}+\boldsymbol{F}_{k} \boldsymbol{f}_{k}+\boldsymbol{G}_{k} \boldsymbol{\omega}_{k} \\
\boldsymbol{y}_{k}=\boldsymbol{H}_{k} \boldsymbol{x}_{k}+\boldsymbol{\nu}_{k}
\end{array}\right.
$$

按照文献 $[4]$ 提出可观测性检验方法, 验证了 (7) 和 (8) 组成的滤波模型是可观测的, 即可以通过观测 来获得状态估计。故障建模成随机游走模型 ${ }^{[8]}$ :

$$
f_{k+1}=f_{k}+\omega_{k}^{f}
$$

通过离散化得到滤波相关矩阵 $\boldsymbol{\Phi}_{k}, \boldsymbol{B}_{k}, \boldsymbol{F}_{k}, \boldsymbol{G}_{k}, \boldsymbol{H}_{k}$, 并根据系统方程中所需传感器先验信息 $q$, 根据以 下公式 ${ }^{[9]}$ 得到滤波所需要的 $Q_{k}^{x}$ :

$$
\left\{\begin{array}{l}
\overline{\boldsymbol{Q}}^{x}=\boldsymbol{G}\left(t_{k}\right) q \boldsymbol{G}\left(t_{k}\right)^{\mathrm{T}} \\
\boldsymbol{M}_{1}=\overline{\boldsymbol{Q}}^{x} \\
\boldsymbol{M}_{i+1}=\boldsymbol{A}\left(t_{k}\right) \boldsymbol{M}_{i}+\left(\boldsymbol{A}\left(t_{k}\right) M_{i}\right)^{\mathrm{T}} \\
\boldsymbol{Q}_{k}^{x}=\sum_{i=1}^{\infty} M_{i} \frac{T^{i}}{i !}
\end{array}\right.
$$

利用此公式计算 $\boldsymbol{Q}_{k}^{x}$ 矩阵, 可以保证 $\boldsymbol{Q}_{k}^{x}$ 矩阵的精度 和正定性, 可以有效防止因为矩阵奇异而导致滤波 中止。

\section{3 最优二步无迹卡尔曼滤波}

\section{1 无迹卡尔曼滤波基本原理}

线性系统经过离散化处理,可以用以下离散系 统描述:

$$
\left\{\begin{array}{l}
\boldsymbol{x}_{k+1}=\boldsymbol{\Phi}_{k} \boldsymbol{x}_{k}+\boldsymbol{B}_{k} \boldsymbol{u}_{k}+\boldsymbol{G}_{k} \boldsymbol{\omega}_{k} \\
\boldsymbol{y}_{k}=\boldsymbol{H}_{k} \boldsymbol{x}_{k}+\boldsymbol{\nu}_{k}
\end{array}\right.
$$

针对上述系统, 卡尔曼滤波的标准形式:

$$
\left\{\begin{array}{l}
\boldsymbol{x}_{k+1 \mid k}=\boldsymbol{\Phi}_{k} \boldsymbol{x}_{k \mid k}+\boldsymbol{B}_{k} \boldsymbol{u}_{k} \\
\boldsymbol{P}_{k+1 \mid k}=\boldsymbol{\Phi}_{k} \boldsymbol{P}_{k \mid k} \boldsymbol{\Phi}_{k}^{\mathrm{T}}+\boldsymbol{Q}_{k} \\
i_{n n o_{k+1}}=\boldsymbol{y}_{k+1}-\boldsymbol{H}_{k+1} \boldsymbol{x}_{k+11 k} \\
\boldsymbol{K}_{k+1}=\boldsymbol{P}_{k+1 \mid k} \boldsymbol{H}_{k+1}^{\mathrm{T}}\left(\boldsymbol{H}_{k+1} \boldsymbol{P}_{k+1 \mid k} \boldsymbol{H}_{k+1}^{\mathrm{T}}+\boldsymbol{R}_{k+1}\right)^{-1} \\
\boldsymbol{x}_{k+1 \mid k+1}=\boldsymbol{x}_{k+1 \mid k}+\boldsymbol{K}_{k+1} \cdot \text { inno }_{k+1} \\
\boldsymbol{P}_{k+1 \mid k+1}=\left(\boldsymbol{I}-\boldsymbol{K}_{k+1} \boldsymbol{H}_{k+1}\right) \boldsymbol{P}_{k+1 \mid k}
\end{array}\right.
$$

以上滤波算法确保了状态估计的最优性,但是系统 (14) 描述的是线性系统, 而现实中的绝大多数系统 都是非线性系统,例如本文所研究的飞行器是一个 典型的复杂非线性系统, 上述公式无法保证滤波结 果的最优性。在文献 [10] 中, 证明了: 


$$
\left\{\begin{array}{l}
\boldsymbol{P}_{k+11 k}^{x y}=\boldsymbol{P}_{k+11 k} \boldsymbol{H}_{k+1}^{\mathrm{T}} \\
\boldsymbol{P}_{k+11 k}^{y y}=\boldsymbol{H}_{k+1} \boldsymbol{P}_{k+1 \mid k} \boldsymbol{H}_{k+1}^{\mathrm{T}}+\boldsymbol{R}_{k+1} \\
\boldsymbol{K}_{k+1}=\boldsymbol{P}_{k+11 k}^{x y}\left(\boldsymbol{P}_{k+11 k}^{y y}\right)^{-1} \\
\boldsymbol{P}_{k+1 \mid k+1}=\boldsymbol{P}_{k+1 \mid k}-\boldsymbol{P}_{k+1 \mid k}^{x y}\left(\boldsymbol{P}_{k+11 k}^{y y}\right)^{-1}\left(\boldsymbol{P}_{k+1 \mid k}^{x y}\right)^{\mathrm{T}}
\end{array}\right.
$$

利用 sigma 点经过非线性 UT 变换 ${ }^{[11]}$ 来传递状态的 均值和方差并利用以上等价形式进行迭代状态估 计, 是 UKF 的基本思想, 并且文献 [12]证明了噪声 高斯分布情况下, UKF 至少保证了状态估计达到 $\mathrm{EKF}$ 三阶截断的精度, 而状态估计协方差矩阵也可 以达到 $\mathrm{EKF}$ 二阶截断精度。UKF 是一个比 $\mathrm{EKF}$ 性 能更优秀的适用于复杂非线性系统的滤波算法。

\section{2 二步卡尔曼滤波基本原理}

本文研究的系统如 (10) 所示, 经过离散化如 (11) 所示, 由于故障 $f$ 的存在, 传统的卡尔曼滤波无 法适用。传感器输出中, 偏差是广泛存在且对精度 影响最大的一种误差。二步卡尔曼滤波器的基本思 想是设计一个无偏差影响的卡尔曼滤波器和一个偏 差滤波器, 并通过耦合矩阵 $V$ 进行耦合实现最终的 状态估计。此文中, 将传感器故障 $f$ 作为一种广义 偏差进行研究,下文中将以故障替代偏差进行阐述。

\section{1) 无故障滤波器基本原理:}

本文将故障建模成了随机游走模型, 考虑到故 障的噪声对无故障滤波器的影响, 对无故障滤波器 的一步预测进行以下补偿:

$$
\begin{aligned}
& x_{k+1 \mid k}^{n f}=x_{k+1 \mid k}^{n f-}+\bar{u}_{k}^{\mathrm{OPT}} \\
& P_{k+1 \mid k}^{n f}=P_{k+1 \mid k}^{n f-}+\bar{Q}_{k}^{\mathrm{OPT}}
\end{aligned}
$$

二步卡尔曼滤波器通过耦合矩阵 $\boldsymbol{V}_{k}$ 和故障滤波器 的输出来计算 $\bar{u}_{k}^{\mathrm{OPT}}, \bar{Q}_{k}^{\mathrm{OPT}}$, 计算的结果确保了状态估 计的最优性。而无故障滤波器的新息, 将作为故障 滤波器的输人。

$$
\text { inno_ } x_{k+1}^{n f}=y_{k+11 k+1}-y_{k+11 k}^{n f}
$$

将作为故障滤波器的输人。

2) 故障滤波器基本原理:

文献 [2]中推导了无故障滤波器的新息和增益 及更新公式:

$$
\left\{\begin{array}{c}
\text { inno_ } f_{k+1}=y_{k+1 \mid k+1}-H_{k+1} x_{k+11 k}^{e s t}= \\
y_{k+1 \mid k+1}-H_{k+1} x_{k+11 k}^{n f}-H_{k+1} U_{k+1} f_{k+1 \mid k}= \\
\quad \text { inno_ } x_{k+1}^{n f}-S_{k+1} f_{k+1 \mid k} \\
K_{k+1}^{f}=P_{k+1 \mid k}^{f} S_{k+1}^{\mathrm{T}}\left(H_{k+1} P_{k+1 \mid k}^{n f} H_{k+1}^{\mathrm{T}}+\right. \\
\left.\quad S_{k+1} P_{k+1 \mid k}^{f} S_{k+1}^{\mathrm{T}}+R\right)^{-1} \\
f_{k+1 \mid k+1}=f_{k+1 \mid k}+K_{k+1}^{f} \cdot i n n o_{-} f_{k+1} \\
P_{k+11 k+1}^{f}=\left(I-K_{k+1}^{f} S_{k+1}\right) P_{k+1 \mid k}^{f}
\end{array}\right.
$$

通过上述公式, 故障滤波器的输人即是无故障滤波 器的新息, 而 $S_{k+1}$ 可以看成是故障滤波器的观测矩 阵。值得注意的是, 故障滤波器的输出即可以实现 IMU 的故障诊断。

3) 耦合矩阵计算与状态估计:

通过下述公式,矩阵 $\boldsymbol{V}$ 可以实现无故障滤波器 的输出和故障滤波器的输出的耦合, 实现飞行器状 态估计：

$$
\left\{\begin{array}{l}
\boldsymbol{V}_{k+1}=\boldsymbol{U}_{k+1}-\boldsymbol{K}_{k+1}^{x_{n f}} \boldsymbol{S}_{k+1} \\
\boldsymbol{x}_{k+1 \mid k+1}^{\text {est }}=\boldsymbol{x}_{k+1 \mid k+1}^{n f}+\boldsymbol{V}_{k+1} \boldsymbol{f}_{k+1 \mid k+1} \\
\boldsymbol{P}_{k+1 \mid k+1}^{\text {est }}=\boldsymbol{P}_{k+1 \mid k+1}^{n f}+\boldsymbol{V}_{k+1} \boldsymbol{P}_{k+1 \mid k+1}^{f} \boldsymbol{V}_{k+1}^{\mathrm{T}}
\end{array}\right.
$$

\section{3 最优二步无迹卡尔曼滤波基本原理}

本文提出了适用于飞行器 IMU 故障诊断的最 优二步无迹卡尔曼滤波算法,算法的具体流程如附 录所示, 滤波相关矩阵可以通过第二部分介绍的离 散化过程获取。为了公式编排, 做了以下简写:

$$
\begin{gathered}
X_{k \mid k}^{\text {sigma }}=\operatorname{sigma}\left(x_{k \mid k}^{n f}, P_{k \mid k}^{n f}\right) \\
X_{k+1 \mid k}^{\text {sigma }}=\operatorname{sigma}\left(x_{k+1 \mid k}^{n f}, P_{k+1 \mid k}^{n f}\right)
\end{gathered}
$$

表示无故障滤波器滤波过程中的 sigma 点生成。

$$
\begin{gathered}
{\left[x_{k+1 \mid k}^{n f-}, P_{k+1 \mid k}^{n f-}\right]=f_{-} U T\left(X_{k \mid k}^{\text {sigma }}\right)} \\
{\left[y_{k+1 \mid k}^{n f}, P_{k+1 \mid k}^{x y}, P_{k+1 \mid k}^{y y}\right]=h_{-} U T\left(X_{k+1 \mid k}^{\text {sigma }}\right)}
\end{gathered}
$$

表示 sigma 点分别经过系统方程和观测方程的 UT 变换。 sigma 点生成和 UT 变换的具体算法实现可 以参考文献 $[13]$ 。

\section{4 实际飞行数据验证与结果分析}

\section{1 实际飞行数据验证}

由于数字仿真环境很难考虑到实际环境的外部 因素干扰,本文采用荷兰代尔夫特理工大学提供的 实际飞行数据 (传感器无故障) 进行验证。风速在 机体系的投影如图 1 所示, 本文所提出的最优二步 无迹卡尔曼滤波方法在风场下仍可以实现飞控系统 IMU 的故障诊断, 验证了所提出方法具有较强的鲁 
棒性。并与基于迭代最优二步扩展卡尔曼滤波 (IOTSEKF) 方法 ${ }^{[14]}$ 进行了对比验证, 说明本文提出 的 OTSUKF 方法具有更加优越的性能。

MATLAB 环境下, 在 $10 \sim 20 \mathrm{~s}, 25 \sim 35 \mathrm{~s}, 40 \sim 50$ $\mathrm{s}$ 分别给加速度计和陀螺仪人为注人阶梯故障 $\mathrm{F} 1$ 、 斜坡故障 F2 和正弦故障 F3 3 种典型故障。

表 1 加速度计和陀螺仪典型故障注入

\begin{tabular}{cccc}
\hline$f$ & \multicolumn{3}{c}{$t$} \\
\cline { 2 - 4 } & $10 \sim 20 \mathrm{~s}$ & $25 \sim 35 \mathrm{~s}$ & $40 \sim 50 \mathrm{~s}$ \\
\hline$f_{a x}$ & $\mathrm{~F} 1$ & $-\mathrm{F} 2$ & $\mathrm{~F} 3$ \\
$f_{a y}$ & $\mathrm{~F} 2$ & $-\mathrm{F} 3$ & $\mathrm{~F} 1$ \\
$f_{a z}$ & $\mathrm{~F} 3$ & $-\mathrm{F} 1$ & $\mathrm{~F} 2$ \\
$f_{p}$ & $\mathrm{~F} 1$ & $-\mathrm{F} 2$ & $\mathrm{~F} 3$ \\
$f_{q}$ & $\mathrm{~F} 2$ & $-\mathrm{F} 3$ & $\mathrm{~F} 1$ \\
$f_{r}$ & $\mathrm{~F} 3$ & $-\mathrm{F} 1$ & $\mathrm{~F} 2$ \\
\hline
\end{tabular}

其中故障的数学解析式为:

$$
\left\{\begin{array}{l}
F 1=1 \\
F 2=0.1 *\left(t-t_{0}\right) \\
F 3=\sin \left[0.5 \pi *\left(t-t_{0}\right)\right]
\end{array}\right.
$$

OTSUKF 的初始化数据如公式 (27) 所示, 其中

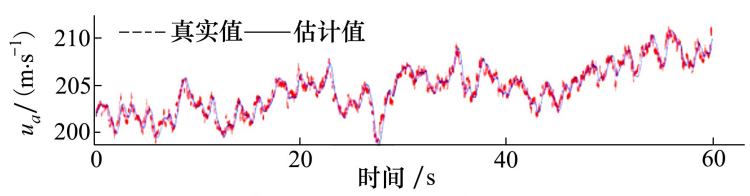

a) 地速在机体系 $x$ 轴的投影

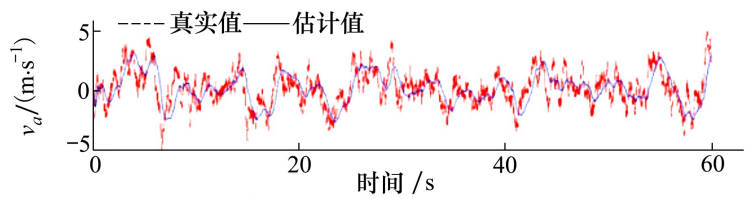

b) 地速在机体系 $y$ 轴的投影

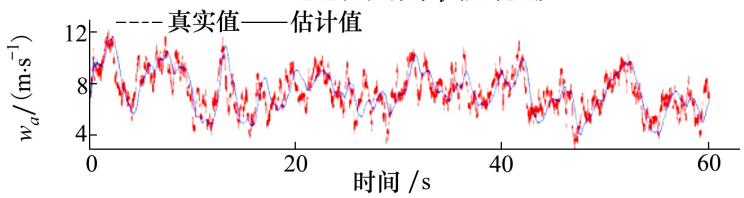

c) 地速在机体系 $z$ 轴的投影

图 1 地速在机体系的投影及 IOTSEKF 的估计值 $q$ 和 $R$ 的特性由 IMU 和 GNSS 的误差特性决定:

$$
\begin{aligned}
& \boldsymbol{x}_{0 \mid 0}=[u(1), v(1), w(1), \varphi(1), \theta(1), \psi(1)]^{\mathrm{T}} \\
& \boldsymbol{P}_{0 \mid 0}^{x}=\operatorname{diag}(1,1,1,1,1,1) \\
& f_{0 \mid 0}=\operatorname{diag}\left(1 \times 10^{-3}, 1 \times 10^{-3}, 1 \times 10^{-3}\right. \text {, } \\
& \left.1 \times 10^{-3}, 1 \times 10^{-3}, 1 \times 10^{-3}\right) \\
& \boldsymbol{P}_{0 \mid 0}^{f}=\operatorname{diag}(1,1,1,1,1,1) \\
& \boldsymbol{P}_{0 \mid 0}^{x f}=\operatorname{diag}\left(1 \times 10^{-6}, 1 \times 10^{-6}, 1 \times 10^{-6}\right. \text {, } \\
& \left.1 \times 10^{-6}, 1 \times 10^{-6}, 1 \times 10^{-6}\right) \\
& \boldsymbol{q}=\operatorname{diag}\left(1 \times 10^{-4}, 1 \times 10^{-4}, 1 \times 10^{-4},\right. \\
& \left.3 \times 10^{-8}, 3 \times 10^{-8}, 3 \times 10^{-8}\right) \\
& \boldsymbol{R}=\operatorname{diag}\left(1 \times 10^{-4}, 1 \times 10^{-4}, 1 \times 10^{-4}\right. \text {, } \\
& \left.3 \times 10^{-8}, 3 \times 10^{-8}, 3 \times 10^{-8}\right) \\
& Q^{f}=\operatorname{diag}\left(1 \times 10^{-4}, 1 \times 10^{-4}, 1 \times 10^{-4}\right. \text {, } \\
& \left.3 \times 10^{-8}, 3 \times 10^{-8}, 3 \times 10^{-8}\right) \\
& \boldsymbol{Q}^{x f}=\operatorname{diag}(0,0,0,0,0,0) \\
& \boldsymbol{V}_{0}=\boldsymbol{P}_{0 \mid 0}^{x f}\left(\boldsymbol{P}_{0 \mid 0}^{f}\right)^{-1} \\
& \begin{array}{l}
\boldsymbol{x}_{0 \mid 0}^{n f}=\boldsymbol{x}_{0 \mid 0}-\boldsymbol{V}_{0} f_{0 \mid 0} \\
\boldsymbol{P}_{0 \mid 0}^{n f}=\boldsymbol{P}_{0 \mid 0}^{x}-\boldsymbol{V}_{0} \boldsymbol{P}_{0 \mid 0}^{f} \boldsymbol{V}_{0}^{\mathrm{T}}
\end{array}
\end{aligned}
$$

状态与故障的实际值和 OTSUKF 的估计值如图 2 5 所示:

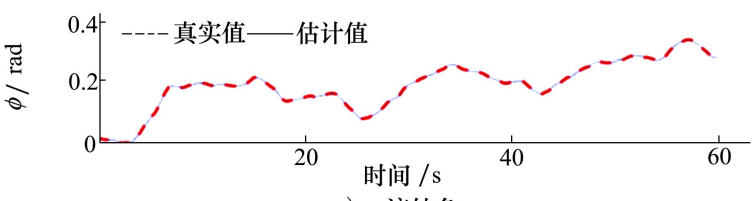

a) 滚转角

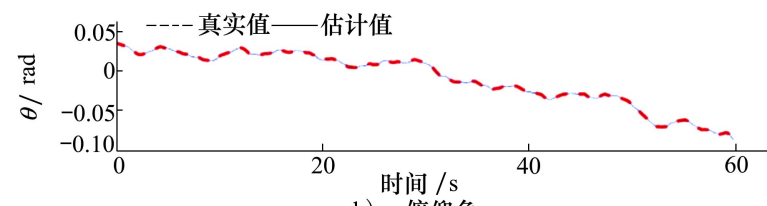

b) 俯仰角

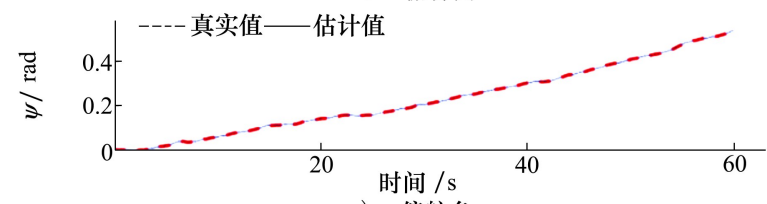

c) 偏航角

图 2 姿态角及 IOTSEKF 的估计值 


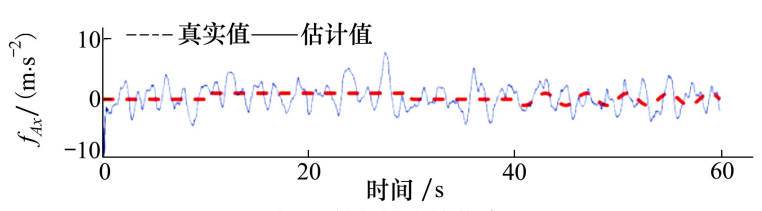

a) $x$ 轴加速度计故障

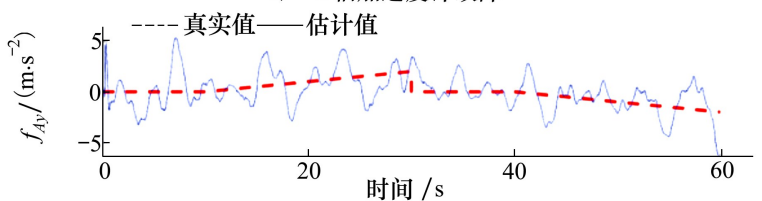

b) $y$ 轴加速度计故障

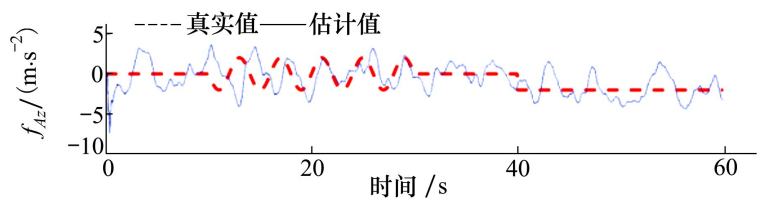

c) $z$ 轴加速度计故障

图 3 加速度计故障及 IOTSEKF 的估计值

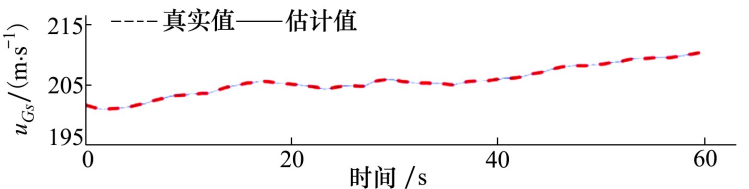

a) 地速在机体系 $x$ 轴的投影

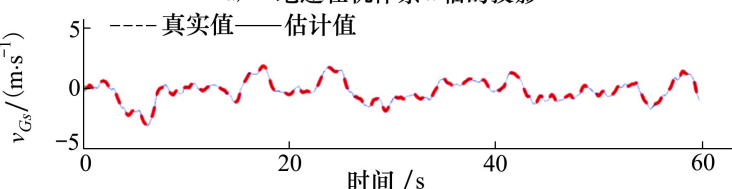

b) 地速在机体系 $y$ 轴的投影

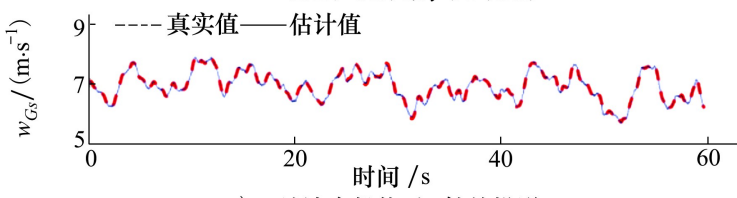

c）地速在机体系 $z$ 轴的投影

图 5 地速在机体系的投影及 OTSUKF 的估计值

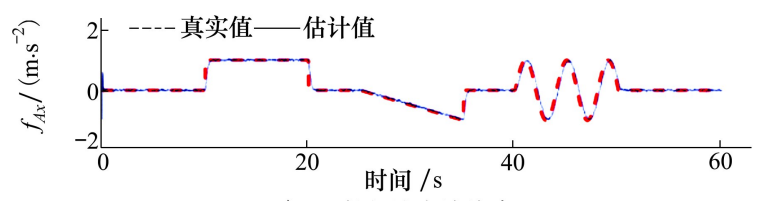

a) $x$ 轴加速度计故障

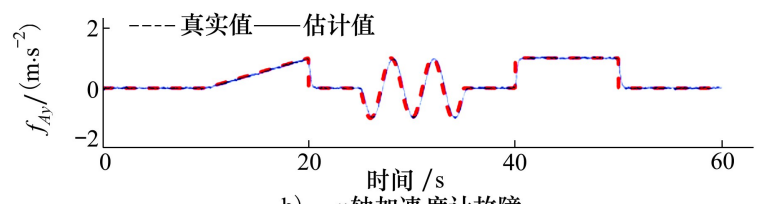

b) $y$ 轴加速度计故障

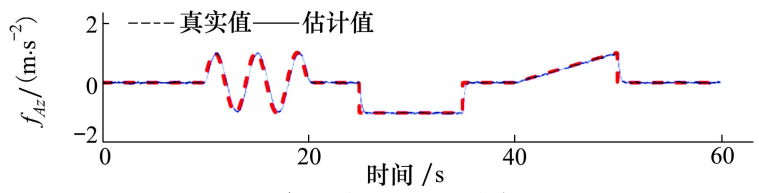

c） $z$ 轴加速度计故障

图 7 加速度计故障及 OTSUKF 的估计值

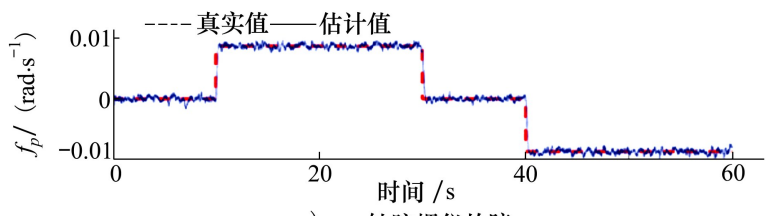

a) $x$ 轴陀螺仪故障

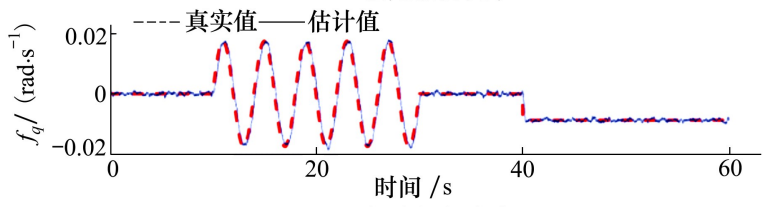

b) $y$ 轴陀螺仪故障

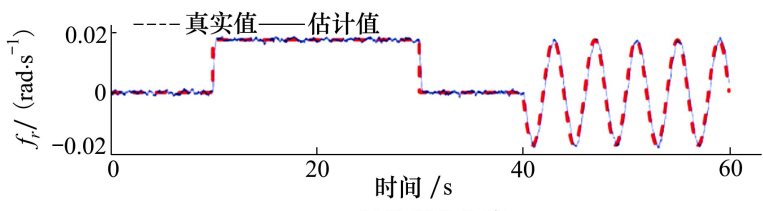

c) $z$ 轴陀螺仪故障

图 4 陀螺仪故障及 IOTSEKF 的估计值

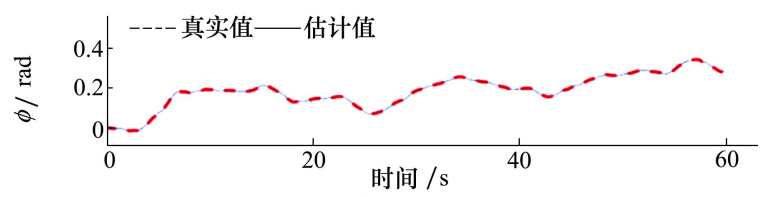

a) 滚转角

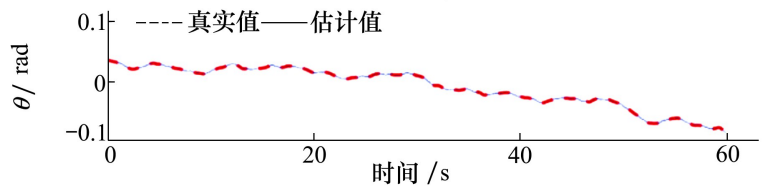

b) 俯仰角

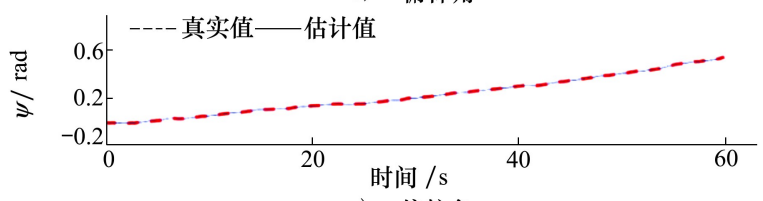

c) 偏航角

图 6 姿态角及 OTSUKF 的估计值

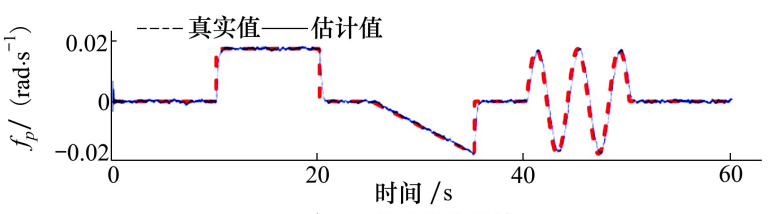

a) $x$ 轴陀螺仪故障

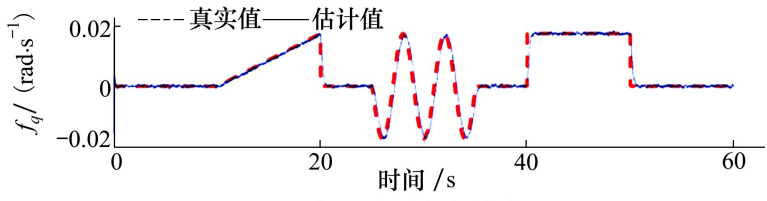

b) $y$ 轴陀螺仪故障

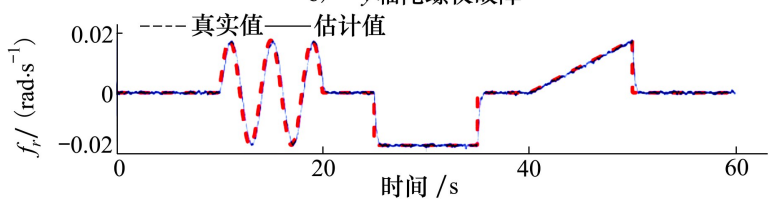

c) $z$ 轴陀螺仪故障

图 8 陀螺仪故障及 OTSUKF 的估计值 


\section{2 结果分析}

基于 IOTSEKF 的 IMU 故障诊断方法所选取的 滤波模型的系统方程与 OTSUKF 方法的系统方程一 致,但是 IOTSEKF 的观测方程为:

$$
\left\{\begin{array}{l}
V_{m}=\sqrt{u^{2}+v^{2}+w^{2}}+v_{V_{a}} \\
\alpha_{m}=\arctan \left(\frac{w}{u}\right)+v_{\alpha} \\
\beta_{m}=\arcsin \left(\frac{v}{V_{a}}\right)+v_{\beta} \\
\varphi_{m}=\varphi+\nu_{\varphi} \\
\theta_{m}=\theta+\nu_{\theta} \\
\psi_{m}=\psi+\nu_{\psi}
\end{array}\right.
$$

式中, $V_{m}, \alpha_{m}, \beta_{m}$ 是真空速、迎角和侧滑角传感器的 测量值。IOTSEKF 的滤波模型没有考虑风扰动的 影响。实际飞行数据中记录的三轴风扰动 $u_{w}, v_{w}$, $w_{w}$ 信息如图 9 所示:

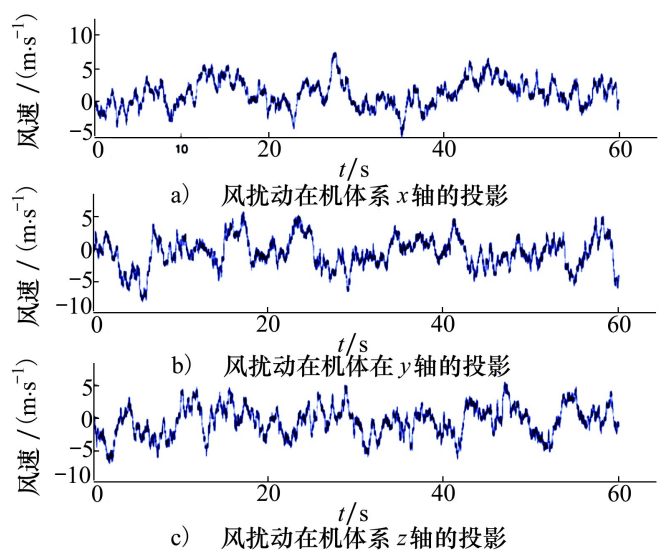

图 9 风扰动在机体系的投影

风扰动存在情况下的观测方程的真实解析关 系为:

$$
\left\{\begin{array}{l}
V_{m}=\sqrt{\left(u-u_{w}\right)^{2}+\left(v-v_{w}\right)^{2}+\left(w-w_{w}\right)^{2}}+v_{V_{a}} \\
\alpha_{m}=\arctan \left(\frac{w-w_{w}}{u-u_{w}}\right)+v_{\alpha} \\
\beta_{m}=\arcsin \left(\frac{v-v_{w}}{V_{a}}\right)+v_{\beta} \\
\varphi_{m}=\varphi+\nu_{\varphi} \\
\theta_{m}=\theta+\nu_{\theta} \\
\psi_{m}=\psi+\nu_{\psi}
\end{array}\right.
$$

风扰动的存在, IOTSEKF 方法的滤波模型存在不确
定性,这会导致滤波器状态估计性能的下降和故障 诊断的不准确。本文修改观测方程如 (8) 式所示, 它在风扰动情况下严格成立, 进而使用 OTSUKF 进 行最优状态估计与故障诊断。

对比图 1 与图 5, 不难发现对于飞行器三轴速 度, OTSUKF 较 IOTSEKF 的状态估计的准确度具有 较大提升。对比图 3 与图 7, 不难发现对于三轴加 速度计的故障诊断, OTSUKF 较 IOTSEKF 的故障诊 断的准确度具有较大提升。这也反映了 OTSUKF 方 法对于风扰动具有鲁棒性,而 IOTSUKF 方法在风扰 动出来情况下, 状态估计和故障诊断效果较差。

由于风扰动主要影响前 3 个观测量, 对于姿态 角的估计和陀螺仪的故障诊断影响较小, 很难分别 从图 2 与图 6 的对比和图 4 与图 8 的对比得到 OTSUKF 较 IOTSEKF 的精确度提升。本文引人一项关 于衡量估计准确度的指标, 均方误差 (root mean square error), 它的定义为:

$$
R M S E=\sqrt{\frac{1}{N}\left(\sum_{i=1}^{N} \hat{x}_{i}-x\right)^{2}}
$$

\begin{tabular}{|c|c|c|c|c|c|}
\hline $\begin{array}{c}\text { RMSE } \\
\text { ( STATE) }\end{array}$ & $\begin{array}{l}\text { IOTS } \\
\text { EKF }\end{array}$ & $\begin{array}{l}\text { OTS } \\
\text { UKF }\end{array}$ & $\begin{array}{c}\text { RMSE } \\
\text { ( FAULT) }\end{array}$ & $\begin{array}{l}\text { IOTS } \\
\text { EKF }\end{array}$ & $\begin{array}{l}\text { OTS } \\
\text { UKF }\end{array}$ \\
\hline$u$ & 0.6930 & 0.0055 & $f_{a x}$ & 1.8469 & 0.1087 \\
\hline$v$ & 1.0580 & 0.0201 & $f_{a y}$ & 1.4372 & 0.1032 \\
\hline$w$ & 1.0565 & 0.0221 & $f_{a z}$ & 1.3718 & 0.1041 \\
\hline$\varphi$ & 0.0057 & 0.0054 & $f_{p}$ & 0.1035 & 0.0854 \\
\hline$\theta$ & 0.0066 & 0.0055 & $f_{q}$ & 0.0876 & 0.0852 \\
\hline$\psi$ & 0.0066 & 0.0058 & $f_{r}$ & 0.1036 & 0.0861 \\
\hline
\end{tabular}

对实际飞行数据验证的数据进行处理, 得到 RMSE 如表 2 所示。分析表 2 的数据, 可以发现风扰动下, OTSUKF 较 IOTSEKF 对于三轴速度的估计的精度 具有较大幅度提升; 对于姿态角的估计精度提升不 明显; 对于加速度计故障诊断的精度具有较大幅度 的提升; 对于陀螺仪故障诊断的精度提升不明显。

表 2 状态估计与故障诊断的均方误差

\section{5 结 论}

本文将二步滤波思想与 UKF 滤波方法结合, 提 出了 OTSUKF 滤波方法。针对于飞行器, 本文通过 创新性的滤波模型设计, 提出了一种适用于飞行器 的滤波模型建立方法,并应用所提出的 OTSUKF 算 
法实现了飞飞行器三轴速度、姿态角的估计与 IMU 传感器故障的诊断。所以提出的 OTSUKF 方法经过 了实际飞行数据验证其对风扰动具有鲁棒性并且与 IOTSEKF 方法进行了对比验证其最优性。所提出
OTSUKF 方法可以实现:

- 对飞行器的速度与姿态信息进行估计。

- 对 IMU 的多种变化类型的故障进行诊断。

- 对风场具有鲁棒性, 可以实际应用。

\section{参考文献:}

[1] Friedland B. Treatment of Bias in Recursive Filtering[J]. IEEE Trans on Automatic Control, 1969, 14(4): 359-367

[2] Alouani A T, Xia P, Rice T R, et al. On the Optimality of Two-Stage State Estimation in the Presence of Random Bias [J]. IEEE Trans on Automatic Control, 1993, 38(8): 1279-1283

[3] Hsieh C S, Chen F C. Optimal Solution of the Two-Stage Kalman Estimator[J]. IEEE Trans on Automatic Control, 1999, 44 (1) : 194-199

[4] He Qizhi, Zhang Weiguo, Liu Xiaoxiong, et al. Information Fusion and Reconstruction of Key Sensors in a Flight Control System in Constant Wind Field Based on Two Stage EKF [C]//Guidance, Navigation and Control Conference, 2016: 718-724

[5] Sonneveldt L. Nonlinear F-16 Model Description[D]. Delft University of Technology, Netherlands, 2006

[6] Stevens B L, Lewis F L, Johnson E N. Aircraft Control and Simulation: Dynamics, Controls Design, and Autonomous Systems [M]. New York, John Wiley \& Sons, 2015

[7] Groves P D. Principles of GNSS, Inertial, and Multisensor Integrated Navigation Systems[ M]. London, Artech House, 2013

[8] Lu P, Van Kampen E J, De Visser C C, et al. Framework for State and Unknown Input Estimation of Linear Time-Varying Systems $[\mathrm{J}]$. Automatica, 2016, 73: 145-154

[9] 秦永元. 卡尔曼滤波与组合导航原理 $[M]$. 西安:西北工业大学出版社, 2015

Qin Yongyuan. The Principle of Kalman Filter and Integrated Navigation [M]. Xi' an, Northwestern Polytechnical University Press, 2015 (in Chinese)

[10] Simon D. Optimal State Estimation: Kalman, H Infinity, And Nonlinear Approaches [M]. New York, Newyork John Wiley \& Sons, 2006

[11] Julier S J, Uhlmann J K. A New Extension of the Kalman Filter to Nonlinear Systems[C]//The 11th International Symposium on Aerospace/Defense Sensing Simulation and Controls, 1997: 110-121

[12] 占荣辉,张军,欧建平, 等. 非线性滤波理论与目标跟踪应用 [M]. 北京: 国防工业出版社,2013

Zhan Ronghui, Zhang Jun, Ou Jianping. Nonlinear Filtering Theory with Target Tracking Application[M]. Beijing, National Defense Industry Press, 2013 (in Chinses)

[13] 黄小平. 卡尔曼滤波原理及应用: Matlab 仿真 $[\mathrm{M}]$. 北京: 电子工业出版社, 2015

Huang Xiaoping. Principles of Kalman Filter and Applications: Matlab Simulation[M]. Beijing, Electronic Industry Press, 2015 (in Chinses)

[14] Lu P, Van Kampen E J. Aircraft Inertial Measurement Unit Fault Identification with Application to Real Flight Data[C] // AIAA Guidance, Navigation, and Control Conference, 2015: 0859 


\title{
Aircraft Inertial Measurement Unit Fault Diagnosis Based on Optimal Two-Stage UKF
}

\author{
He Qizhi ${ }^{1}$, Zhang Weiguo ${ }^{1}$, Huang Degang ${ }^{2}$, Chen Huakun ${ }^{1}$, Liu Jinglong ${ }^{1}$ \\ (1.School of Automation, Northwestern Polytechnical University, Xi' an 710129, China; \\ 2.Aviation Ammunition Research Institute of Weapon Industry Group, Harbin 150036, China)
}

\begin{abstract}
Optimal two stage Kalman filter ( OTSKF) is able to obtain optimal estimation of system states and bias for linear system which contains random bias. Unscented Kalman filter (UKF) is a conventional nonlinear filtering method which utilizes Sigmas point sampling and unscented transformation technology realizes propagation of state means and covariances through nonlinear system. Aircraft is a typical complicate nonlinear system, this paper treats the faults of Inertial Measurement Unit (IMU) as random bias, established a filtering model which contains faults of IMU. Hybird the two stage filtering technique and UKF, this paper proposed an optimal two stage unscented Kalman filter (OTSUKF) algorithm which is suitable for fault diagnosis of IMU, realized optimal estimation of system states and faults identification of IMU via proposed innovative designing method of filtering model and the algorithm was validated that it is robust to wind disterbance via real flight data and it is also validated that proposed OTSUKF is optimal in the existance of wind disturbance via comparing with the existance iterated optimal two stage extended kalman filter (IOTSEKF) method.
\end{abstract}

Keywords: optimal two stage unscented kalman filter; random bias; inertial measurement unit; state estimation; fault diagnosis 